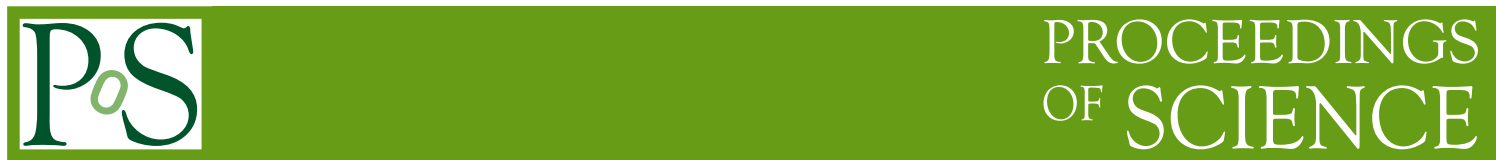

Edinburgh 2008/37

POS (LAT2008) 269

\title{
Neutral Kaon Mixing Beyond the Standard Model from 2+1 Flavour Domain Wall QCD
}

\author{
Jan Wennekers* for the RBC and UKQCD collaborations \\ School of Physics and Astronomy, \\ The University of Edinburgh, \\ Edinburgh EH9 3JZ, UK, \\ E-mail: jwennekeeph.ed.ac.uk
}

\begin{abstract}
We present preliminary results of a study of $\Delta S=2$ matrix elements originating from physics beyond the Standard Model. Using $2+1$ flavour Domain Wall Fermions we obtain the nonperturbative renormalisation (mixing) matrix in the RI scheme. We also discuss plans for the chiral extrapolation of the renormalised matrix elements in a partially quenched set-up.
\end{abstract}

The XXVI International Symposium on Lattice Field Theory

July 14 - 19, 2008

Williamsburg, Virginia, USA

\footnotetext{
* Speaker.
} 


\section{Motivation}

In the Standard Model (SM) there is only one four-quark operator contributing to the analysis of $K_{0}-\bar{K}_{0}$ mixing. It originates from box diagrams with $W$ bosons, Figure 1. In theories beyond the SM there are additional box diagrams. Figure 2 shows an example from the mass insertion approximation of a supersymmetric extension of the SM.

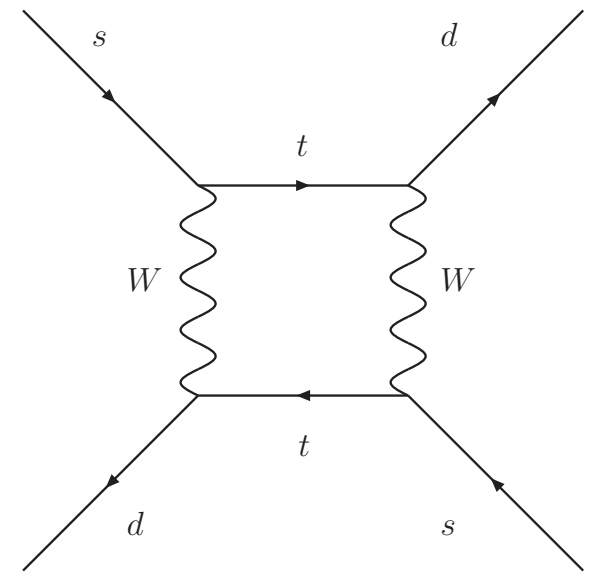

Figure 1: Box diagram for $K_{0}-\bar{K}_{0}$ mixing in the Standard Model.

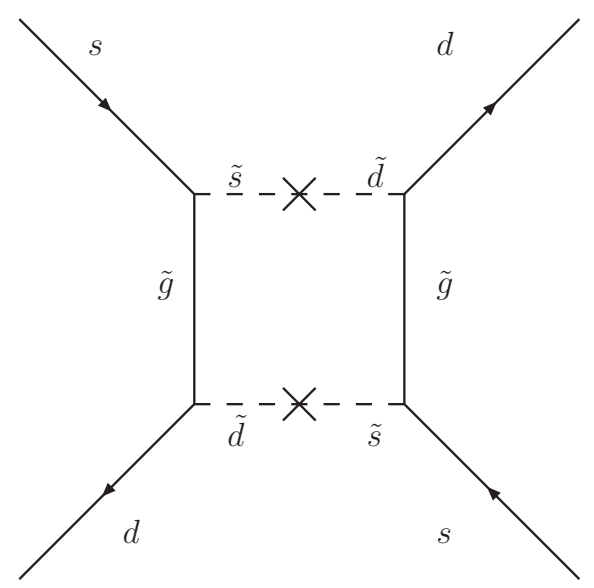

Figure 2: Example for a SUSY box diagram with gluinos and squarks in the mass insertion approximation.

The matrix element of the SM operator

$$
Q_{1}=\bar{s}^{a} \gamma_{\mu} P_{L} d^{a} \bar{s}^{b} \gamma^{\mu} P_{L} d^{b}
$$

is parametrised by the bag parameter $B_{K}$. $Q_{1}$ renormalises multiplicatively for lattice discretisations with chiral symmetry. The most advanced computations of $B_{K}$ have exploited the chiral symmetry of Domain Wall Fermions (DWF) [1] or overlap fermions [2]. The good agreement between the measured value for $|\varepsilon|$ and the SM with lattice $B_{K}$ input puts a constraint on theories beyond the Standard Model (BSM) which allow for additional classes of box diagrams. The lattice can contribute here as well by providing hadronic matrix elements for a basis of four-quark operators which then can be used in phenomenological studies. Again lattice fermions with good chiral symmetry are essential since renormalisation becomes continuum-like.

Besides the SM operator $Q_{1}(1.1)$ we consider the operators,

$$
\begin{aligned}
& Q_{2}=\bar{s}^{a} \gamma_{\mu} P_{L} d^{a} \bar{s}^{b} \gamma^{\mu} P_{R} d^{b}, \\
& Q_{3}=\bar{s}^{a} P_{L} d^{a} \bar{s}^{b} P_{R} d^{b}, \\
& Q_{4}=\bar{s}^{a} P_{L} d^{a} \bar{s}^{b} P_{L} d^{b}, \\
& Q_{5}=\bar{s}^{a} \sigma_{\mu v} P_{L} d^{a} \bar{s}^{b} \sigma^{\mu v} P_{L} d^{b} .
\end{aligned}
$$

This basis is related to the supersymmetric basis used in several other studies by a Fierz transformation. The anomalous dimensions for these operators are known to next-to-leading order $[3,4]$. 
The mixing among the BSM operators is limited to two $2 \times 2$ blocks,

$$
\gamma=\left(\begin{array}{ccccc}
\gamma_{11} & 0 & 0 & 0 & 0 \\
0 & \gamma_{22} & \gamma_{23} & 0 & 0 \\
0 & \gamma_{32} & \gamma_{33} & 0 & 0 \\
0 & 0 & 0 & \gamma_{44} & \gamma_{45} \\
0 & 0 & 0 & \gamma_{54} & \gamma_{55}
\end{array}\right)
$$

\section{Non-perturbative Renormalisation}

To subtract the logarithmic divergence in the matrix elements of the operators $Q_{i}$ we employ the RI scheme [5] at a scale $\mu$. We consider the full $5 \times 5$ matrix,

$$
Q_{i}^{\mathrm{RI}}=Z_{i j}(\mu) Q_{j}
$$

despite the reduced mixing of the continuum theory. Since our lattice action is chirally symmetric up to a small violation of $O\left(m_{\text {res }}\right)$ we expect the elements of $Z$ which vanish in the presence of chiral symmetry to be small. Our approach enables us to check this assumption and to compare the renormalised results with the full matrix and the block diagonal one.

We compute the amputated four-point vertex functions $\Gamma$ and project them onto the relevant Dirac structure. The resulting matrix $\Lambda$ yields the renormalisation matrix,

$$
\frac{1}{Z_{q}^{2}} Z(\mu)=\Lambda_{\text {tree }} \cdot \Lambda^{-1}\left(p^{2}=\mu^{2}\right)
$$

The wave function renormalisation $Z_{q}$ is eliminated using the (local) axial current and its renormalisation constant $Z_{A}$ which can be determined independently from the axial Ward identity for Domain Wall Fermions [6],

$$
\frac{1}{Z_{A}^{2}} Z=\Lambda_{\text {tree }} \cdot \Lambda^{-1} / \Lambda_{A}
$$

The standard RI renormalisation condition is defined at an unfortunate kinematic point. The momenta of all four involved quarks are the same and there is no momentum flowing out of the vertex. This so called exceptional momentum configuration leads to additional spontaneous chiral symmetry breaking from subgraphs which are only suppressed as a small inverse power of momentum. This has been seen in the splitting between $\Lambda_{A}$ and $\Lambda_{V}$, the amputated vertex functions for the axial and vector current [6]. The effect on the renormalisation factors for the operators $Q_{2}$ to $Q_{5}$ is a $\frac{1}{m p^{2}}$ divergence. There are at least two suggestions in the literature how to deal with this pion pole. One can try to directly fit the pole in $\Lambda_{i j}$ [7] or one can form ratios of the type [8]

$$
\frac{m_{1} \Lambda_{i j}\left(m_{1}, p^{2}\right)-m_{2} \Lambda_{i j}\left(m_{2}, p^{2}\right)}{m_{1}-m_{2}}
$$

where $m_{1}$ and $m_{2}$ are two different quark masses. The ratio in the chiral limit corresponds to the subtracted $\Lambda_{i j}$ [9].

We have implemented a slightly different renormalisation condition which directly gives the amputated Green's function without the pion pole. In this non-exceptional case one still has $p_{i}^{2}=$ 
$\mu^{2}$ for all legs of the vertex, but there are two pairs of momenta which differ such that the sum of momenta at the vertex is also $\mu^{2}$. Now chirality breaking subgraphs are suppressed by the relatively high scale.

In Figure 3 we compare the two kinematic set-ups for the row of the matrix $\Lambda$ which determines the mixing for $Q_{2}$. The main difference is the suppression of mixing with the operators $Q_{4}$ and $Q_{5}$ which is caused by the mentioned chirality breaking subgraphs. This clearly shows the advantages of the non-exceptional momentum data. The main obstacle for the use of this new approach is the lack of perturbative results for the matching to perturbative schemes like $\overline{\mathrm{MS}}$. A first result has been presented at this conference for the case of $Z_{m}$ [10]. Such infrared problems not only appear in the non-perturbtative data, but also seem to influence the rate of convergence of continuum perturbation theory, which is very promising for the RI-MOM approach.
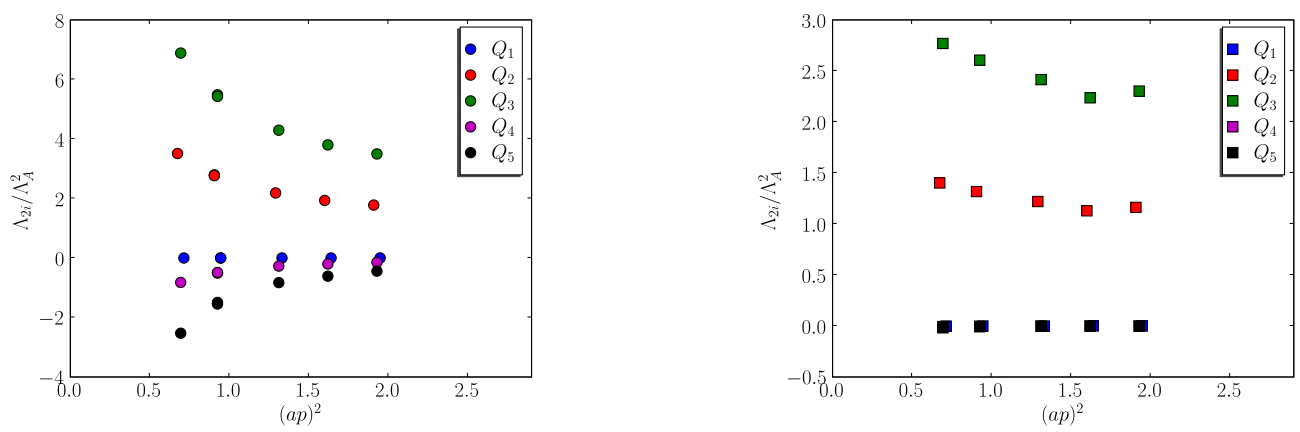

Figure 3: Elements of the matrix $\Lambda / \Lambda_{A}^{2}$ associated with the mixing for operator $Q_{2}$ for a range of $p^{2}$ with exceptional momenta (left) and non-exceptional momenta (right). The statistical errors are smaller than the symbols. The chiral symmetry of DWF relating $Q_{1}$ and $Q_{2}, Q_{3}$ and $Q_{4}$ is manifest in the non-exceptional case, but obscured by spontaneous chiral symmetry breaking at low $p^{2}$ in the conventional approach.

Recently we have adopted the use of gauge-fixed momentum sources which project on a single momentum at the source. The vertex then has to be put at the sink and a full volume average is possible. These sources allow us to reach a better statistical accuracy at much reduced cost especially on large volumes. Even though each momentum requires new propagator inversions the new method scales much better with the lattice volume and has to be the method of choice for our new large lattices.

\section{Results for Bare Matrix Elements}

The preliminary results presented in this section are part of the ongoing project with $32^{3} \times 64 \times$ 32 ensembles of $N_{f}=2+1$ Domain Wall Fermions with the Iwasaki gauge action at $\beta=2.25$ [11].

The SM matrix element is commonly normalised by its value in the vacuum saturation approximation. Using the same normalisation for the other four operators leads to a divergent chiral limit for the resulting bag parameters. This divergence can be moved into the normalisation, but this requires the knowledge of renormalised quark masses for the phenomenological use of the results. 


\begin{tabular}{c|c|c|c|c}
$m_{l}$ & $m_{s}$ & $m_{\pi}$ & Renorm. & Matrix Elements \\
\hline 0.004 & 0.03 & $\sim 300 \mathrm{MeV}$ & 0.004 & $0.002,0.004,0.006,0.008$ \\
& & & & $0.025,0.03$ \\
0.006 & 0.03 & $\sim 365 \mathrm{MeV}$ & 0.006 &, \\
0.008 & 0.03 & $\sim 420 \mathrm{MeV}$ & 0.008 &,
\end{tabular}

Therefore alternative normalisations like are preferable. But for the purpose of this write-up we stick with the $B_{K}$ normalisation since all results are preliminary,

$$
\begin{aligned}
\left\langle\bar{K}\left|Q_{i}\right| K\right\rangle & =N_{i} m_{K}^{2} F_{K}^{2} B_{i}, \quad i=1, \ldots, 5, \\
N_{i} & =\frac{8}{3},-\frac{4}{3} R, 2 R, \frac{5}{3} R,-4 R, \\
R & =\left(\frac{m_{K}}{m_{s}^{r}+m_{d}^{r}}\right)^{2} .
\end{aligned}
$$

Gauge-fixed wall sources have been shown to be a very efficient for $B_{K}[1,12]$. We reach the same level of statistical uncertainty when applying this approach to the whole operator basis. In Figure 7 we give an impression of the quality of the data for the four non-SM operators. Each plot shows the fully dynamical data $\left(m_{\mathrm{val}}=m_{\text {sea }}\right)$ for both light and strange quark mass. With around 100 configurations the statistical errors are of the order of $1 \%$ at the lowest dynamical mass for the bare $B$ parameters.

Our plan for the chiral extrapolation of this data set is again along the lines of the existing work on $B_{K}$. We use an approach based of $S U(2)$ Chiral Perturbation Theory (ChPT). In this setup the leading order Low Energy Constants $F$ and $B_{0}$ are fixed from fits in the pion sector. The kaon is treated as a heavy meson [13]. The needed partially quenched formulae can be derived from results in Heavy Meson ChPT [14]. In Figure 5 we show the light quark mass dependence for BSM operators.

\section{Summary}

We have given a status report on a computation of $\Delta S=2$ matrix elements for a complete operator basis. The aim of this study is to compute the renormalised matrix elements with dynamical fermions to have an impact on phenomenological studies.

The use of Domain Wall Fermions with very small chiral symmetry breaking is essential for the continuum-like renormalisation of the operators. We use the RI scheme with gauge-fixed momentum sources which greatly improves the statistical accuracy of the renormalisation constants. We are exploring a variant of the RI scheme with a non-exceptional momentum configuration to reduce effects from chiral symmetry breaking at low momentum. 

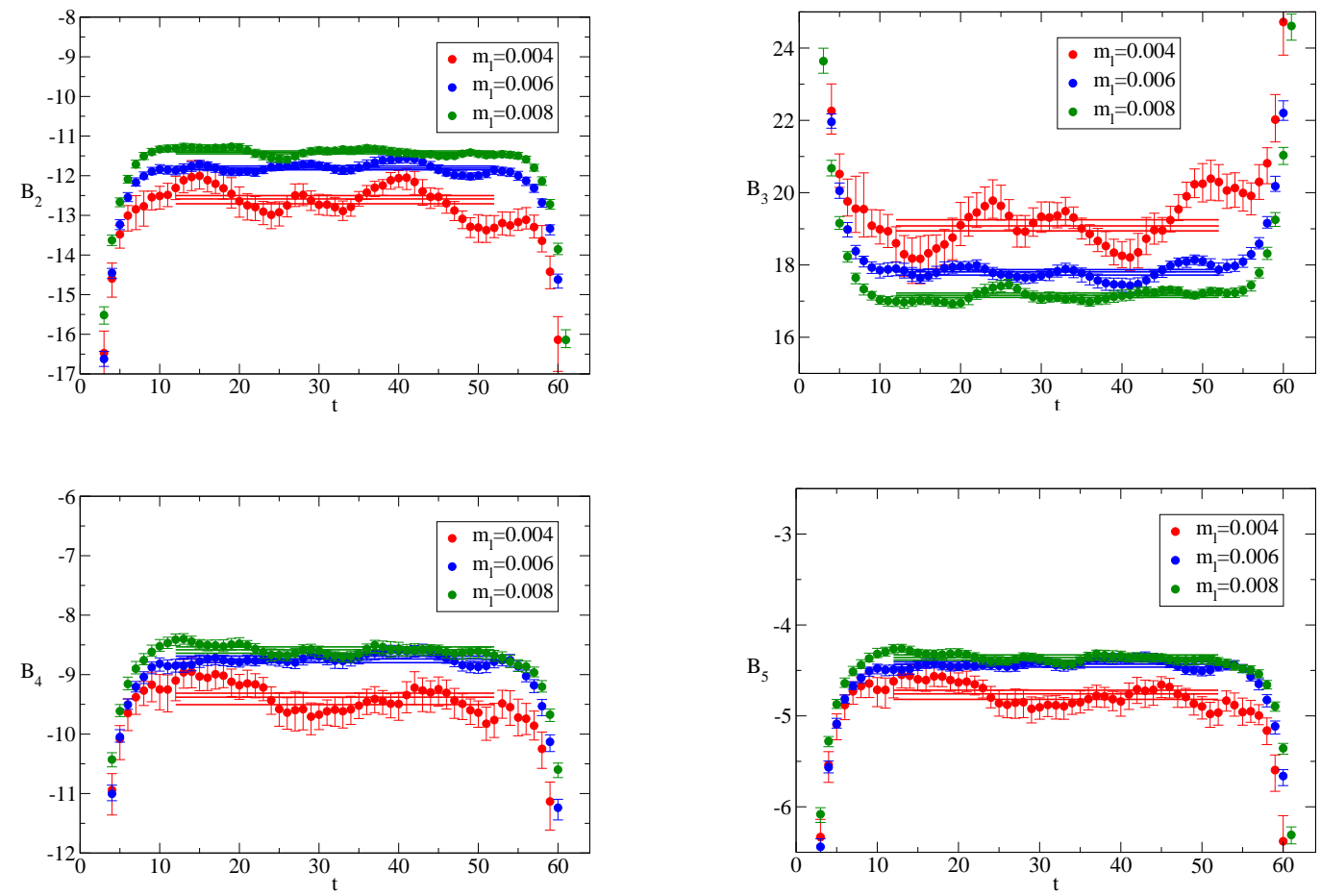

Figure 4: Plateau plots for the ratios $B_{2}-B_{5}$ at the unitary quark masses of all three ensembles without normalisation factor $N_{i}(3.2)$.

We have computed the bare matrix elements on the $32^{3} \times 64 \times 16$ ensembles with small statistical errors for six valence masses. We plan to extrapolate this data using SU(2) Chiral Perturbation Theory in the same way as for $B_{K}$.

We also intend to extend this work to the coarser $24^{3}$ lattices which will allow us to quantify the size of $O\left(a^{2}\right)$ lattice artefacts.

\section{Acknowledgements}

We thank our colleagues in RBC and UKQCD within whose programme this calculation was performed. We thank the QCDOC design team for developing the QCDOC machine and its software. This development and the computers used in this calculation were funded by the U.S.DOE grant DE-FG02-92ER40699, PPARC JIF grant PPA/J/S/1998/0075620 and by RIKEN. This work was supported by DOE grant DE-FG02-92ER40699 and PPARC grants PPA/G/O/2002/00465 and PP/D000238/1. We thank the University of Edinburgh, PPARC, RIKEN, BNL and the U.S. DOE for providing the QCDOC facilities used in this calculation.

\section{References}

[1] RBC and UKQCD Collaboration, D. J. Antonio et al. Phys. Rev. Lett. 100 (2008) 032001 arXiv:hep-ph/0702042]. 

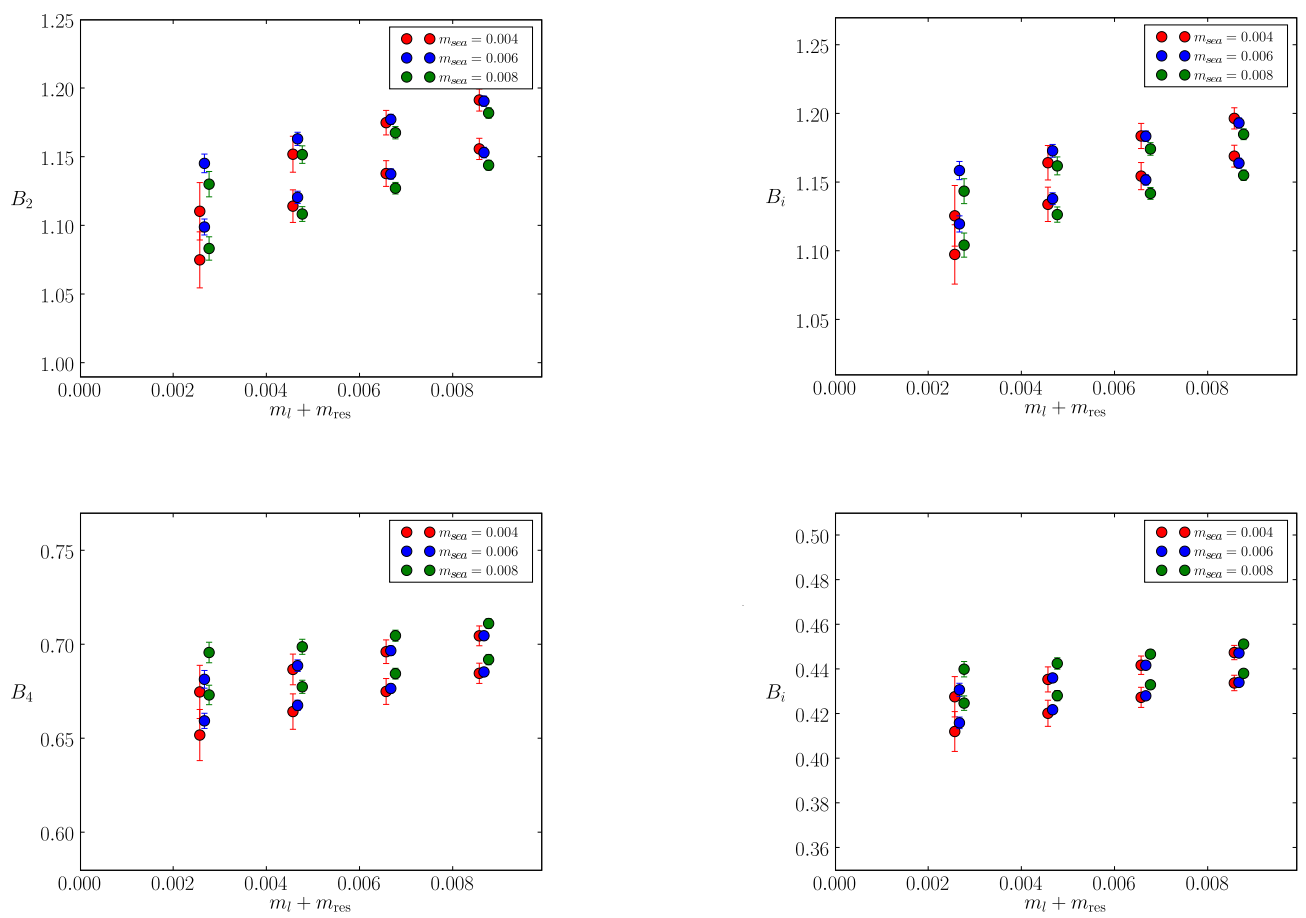

Figure 5: Light quark mass dependence for the bag parameters $B_{2}-B_{5}$. The different colours indicate the three sea quark masses. In each plot there is data for two strange quark masses, 0.025 and 0.03 .

[2] JLQCD Collaboration, S. Aoki et al. Phys. Rev. D77 (2008) 094503 [arXiv : 0801.4186 [hep-lat]].

[3] M. Ciuchini et al. JHEP 10 (1998) 008 [arXiv: hep-ph/9808328].

[4] A. J. Buras, M. Misiak and J. Urban Nucl. Phys. $\mathbf{B 5 8 6}$ (2000) 397-426 [arXiv:hep-ph/0005183].

[5] G. Martinelli, C. Pittori, C. T. Sachrajda, M. Testa and A. Vladikas Nucl. Phys. B445 (1995) 81-108 arXiv:hep-lat/9411010].

[6] RBC and UKQCD Collaboration, Y. Aoki et al. arXiv:0712.1061 [hep-lat].

[7] R. Babich et al. Phys. Rev. D74 (2006) 073009 arXiv: hep-lat/0 605016].

[8] L. Giusti and A. Vladikas Phys. Lett. B488 (2000) 303-312 [arXiv: hep-lat/0005026.

[9] D. Becirevic et al. JHEP 08 (2004) 022 [arXiv: hep-lat/0401033].

[10] RBC and UKQCD Collaboration, Y. Aoki PoS LAT2008 (2008) 222.

[11] RBC and UKQCD Collaboration, E. E. Scholz PoS LAT2008 (2008) 095 [arXiv : 0809 . 3251 [hep-lat]].

[12] RBC and UKQCD Collaboration, C. Kelly PoS LAT2008 (2008) 270.

[13] RBC and UKQCD Collaboration, C. Allton et al. arXiv:0804.0473 [hep-lat].

[14] W. Detmold and C. J. D. Lin Phys. Rev. D76 (2007) 014501 [arXiv: hep-lat/0612028. 